\title{
Improving Forecasting Accuracy in the Case of Intermittent Demand Forecasting
}

\author{
Daisuke Takeyasu ${ }^{1}$ \\ ${ }^{1}$ The Open University of Japan, \\ Chiba City, Japan
}

\author{
Asami Shitara $^{2}$ \\ ${ }^{2}$ Tax Corporation Arknet, Shizuoka \\ City, Japan
}

\author{
Kazuhiro Takeyasu ${ }^{3}$ \\ ${ }^{3}$ College of Business Administration, \\ Tokoha University, Shizuoka, Japan
}

\begin{abstract}
In making forecasting, there are many kinds of data. Stationary time series data are relatively easy to make forecasting but random data are very difficult in its execution for forecasting. Intermittent data are often seen in industries. But it is rather difficult to make forecasting in general. In recent years, the needs for intermittent demand forecasting are increasing because of the constraints of strict Supply Chain Management. How to improve the forecasting accuracy is an important issue. There are many researches made on this. But there are rooms for improvement. In this paper, a new method for cumulative forecasting method is proposed. The data is cumulated and to this cumulated time series, the following method is applied to improve the forecasting accuracy. Trend removing by the combination of linear and $2^{\text {nd }}$ order non-linear function and $3^{\text {rd }}$ order non-linear function is executed to the production data of $\mathrm{X}$ ray image intensifier tube device and Diagnostic $\mathrm{X}$-ray image processing apparatus. The forecasting result is compared with those of the non-cumulative forecasting method. The new method shows that it is useful for the forecasting of intermittent demand data. The effectiveness of this method should be examined in various cases.
\end{abstract}

Keywords-intermittent demand forecasting; minimum variance; exponential smoothing method; trend

\section{INTRODUCTION}

Supply chain management is inevitable in industries in recent years. Demand forecasting is the basis in supply chain management. In industries, how to improve forecasting accuracy such as sales, shipping is an important issue. There are cases that intermittent demand forecasting is required. But the mere application of the past method does not bear good estimation of parameters and exquisite forecasting.

There are many researchers made on this.

Based upon the Croston's model (Box et al.2008), Shenstone and Hyndma (2005) analyzed the intermittent demand forecasting. Troung et al. (2011) applied Neural Network to intermittent demand forecasting. Tanaka et al. (2012) has built sales forecasting model for book publishing, where they have devised cumulative forecasting method.

In this paper, we further develop this cumulative forecasting method in order to improve the forecasting accuracy for intermittent demand.
A new method for cumulative forecasting method is proposed.

The data is cumulated and to this cumulated time series, the following method is applied to improve the forecasting accuracy. Focusing that the equation of exponential smoothing method(ESM) is equivalent to $(1,1)$ order ARMA model equation, a new method of estimation of smoothing constant in exponential smoothing method is proposed before by us which satisfies minimum variance of forecasting error[7]. Generally, smoothing constant is selected arbitrarily. But in this paper, we utilize above stated theoretical solution. Firstly, we make estimation of ARMA model parameter and then estimate smoothing constants.

Thus theoretical solution is derived in a simple way and it may be utilized in various fields. Furthermore, combining the trend removing method with this method, we aim to improve the forecasting accuracy. An approach to this method is executed in the following method. Trend removing by the combination of linear and $2^{\text {nd }}$ order non-linear function and $3^{\text {rd }}$ order non-linear function is executed to the data of X-ray image intensifier tube device and Diagnostic X-ray image processing apparatus. The weights for these functions are set 0.5 for two patterns at first and then varied by 0.01 increment for three patterns and optimal weights are searched. For the comparison, monthly trend is removed after that. Theoretical solution of smoothing constant of ESM is calculated for both of the monthly trend removing data and the non-monthly trend removing data. Then forecasting is executed on these data.

The forecasting result is compared with those of the noncumulative forecasting method. The new method shows that it is useful for the forecasting of intermittent demand data. The effectiveness of this method should be examined in various cases.

The rest of the paper is organized as follows. In section 2, the new method is described. ESM is stated by ARMA model and estimation method of smoothing constant is derived using ARMA model identification. The combination of linear and non-linear function is introduced for trend removing and the Monthly Ratio is also referred. Forecasting is executed in section 3, and estimation accuracy is examined, which is followed by the Discussion of section 4 


\section{DESCRIPTION OF THE NEW METHOD}

\section{A. Description of ESM Using ARMA Model ${ }^{[5]}$}

In ESM, forecasting at time $t+1$ is stated in the following equation.

$$
\begin{array}{r}
\hat{x}_{t+1}=\hat{x}_{t}+\alpha\left(x_{t}-\hat{x}_{t}\right) \\
=\alpha x_{t}+(1-\alpha) \hat{x}_{t}
\end{array}
$$

Here,

$\hat{x}_{t+1}$ : forecasting at $t+1$

$x_{t}$ : realized value at $t$

$\alpha$ : smoothing constant $(0<\alpha<1)$

(1) is re-stated as

$$
\hat{x}_{t+1}=\sum_{l=0}^{\infty} \alpha(1-\alpha)^{l} x_{t-l}
$$

By the way, we consider the following $(1,1)$ order ARMA model.

$$
x_{t}-x_{t-1}=e_{t}-\beta e_{t-1}
$$

Generally, $(p, q)$ order ARMA model is stated as

$$
x_{t}+\sum_{i=1}^{p} a_{i} x_{t-i}=e_{t}+\sum_{j=1}^{q} b_{j} e_{t-j}
$$

Here,

$\left\{x_{t}\right\}$ : Sample process of Stationary Ergodic Gaussian Process $x(t) t=1,2, \cdots, N, \cdots$

\section{$\left\{e_{t}\right\}$ : Gaussian White Noise with 0 mean $\sigma_{e}^{2}$ variance}

MA process in (4) is supposed to satisfy convertibility condition. Utilizing the relation that

$$
E\left[e_{t} \mid e_{t-1}, e_{t-2}, \cdots\right]=0
$$

we get the following equation from (3).

$$
\hat{x}_{t}=x_{t-1}-\beta e_{t-1}
$$

Operating this scheme on $t+1$, we finally get

$$
\begin{aligned}
\hat{x}_{t+1} & =\hat{x}_{t}+(1-\beta) e_{t} \\
& =\hat{x}_{t}+(1-\beta)\left(x_{t}-\hat{x}_{t}\right)
\end{aligned}
$$

If we set $1-\beta=\alpha$, the above equation is the same with (1), i.e., equation of ESM is equivalent to $(1,1)$ order ARMA model, or is said to be $(0,1,1)$ order ARIMA model because 1 st order AR parameter is $-1^{[1]}$.

Comparing with (3) and (4), we obtain

$$
\left\{\begin{array}{l}
a_{1}=-1 \\
b_{1}=-\beta
\end{array}\right.
$$

From (1), (6),

$$
\alpha=1-\beta
$$

Therefore, we get

$$
\left\{\begin{array}{l}
a_{1}=-1 \\
b_{1}=-\beta=\alpha-1
\end{array}\right.
$$

From above, we can get estimation of smoothing constant after we identify the parameter of MA part of ARMA model. But, generally MA part of ARMA model become non-linear equations which are described below. Let (4) be

$$
\begin{aligned}
& \tilde{x}_{t}=x_{t}+\sum_{i=1}^{p} a_{i} x_{t-i} \\
& \tilde{x}_{t}=e_{t}+\sum_{j=1}^{q} b_{j} e_{t-j}
\end{aligned}
$$

We express the autocorrelation function of $\tilde{x}_{t}$ as $\tilde{r}_{k}$ and from (8), (9), we get the following non-linear equations which are well known.

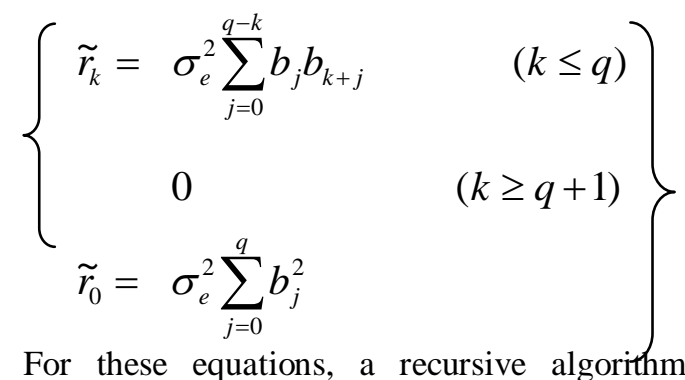

For these equations, a recursive algorithm has been developed. In this paper, parameter to be estimated is only $b_{1}$, so it can be solved in the following way.

From (3) (4) (7) (10), we get

$$
\begin{aligned}
& q=1 \\
& a_{1}=-1 \\
& b_{1}=-\beta=\alpha-1 \\
& \tilde{r}_{0}=\left(1+b_{1}^{2}\right) \sigma_{e}^{2} \\
& \tilde{r}_{1}=b_{1} \sigma_{e}^{2}
\end{aligned}
$$

If we set

$$
\rho_{k}=\frac{\tilde{r}_{k}}{\tilde{r}_{0}}
$$

the following equation is derived.

$$
\rho_{1}=\frac{b_{1}}{1+b_{1}^{2}}
$$

We can get $b_{1}$ as follows.

$$
b_{1}=\frac{1 \pm \sqrt{1-4 \rho_{1}^{2}}}{2 \rho_{1}}
$$


In order to have real roots, $\rho_{1}$ must satisfy

$$
\left|\rho_{1}\right| \leq \frac{1}{2}
$$

From invertibility condition, $b_{1}$ must satisfy

$$
\left|b_{1}\right|<1
$$

From (13), using the next relation,

$$
\begin{aligned}
& \left(1-b_{1}\right)^{2} \geq 0 \\
& \left(1+b_{1}\right)^{2} \geq 0
\end{aligned}
$$

(15) always holds. As

$$
\alpha=b_{1}+1
$$

$b_{1}$ is within the range of

$$
-1<b_{1}<0
$$

Finally we get

$$
\left.\begin{array}{l}
b_{1}=\frac{1-\sqrt{1-4 \rho_{1}^{2}}}{2 \rho_{1}} \\
\alpha=\frac{1+2 \rho_{1}-\sqrt{1-4 \rho_{1}^{2}}}{2 \rho_{1}}
\end{array}\right\}
$$

which satisfy above condition. Thus we can obtain a theoretical solution by a simple way.

Here ${ }^{\rho_{1}}$ must satisfy

$$
-\frac{1}{2}<\rho_{1}<0
$$

in order to satisfy $0<\alpha<1$.

Focusing on the idea that the equation of ESM is equivalent to

$(1,1)$ order ARMA model equation, we can estimate smoothing constant after estimating ARMA model parameter.

It can be estimated only by calculating 0 th and 1 st order autocorrelation function.

\section{B. Trend Removal Method ${ }^{[5]}$}

As trend removal method, we describe the combination of linear and non-linear function.

[1] Linear function

We set

$$
y=a_{1} x+b_{1}
$$

as a linear function.

[2] Non-linear function
We set

$$
\begin{gathered}
y=a_{2} x^{2}+b_{2} x+c_{2} \\
y=a_{3} x^{3}+b_{3} x^{2}+c_{3} x+d_{3}
\end{gathered}
$$

as a $2^{\text {nd }}$ and a $3^{\text {rd }}$ order non-linear function.

[3] The combination of linear and non-linear function We set

$$
\begin{gathered}
y=\alpha_{1}\left(a_{1} x+b_{1}\right)+\alpha_{2}\left(a_{2} x^{2}+b_{2} x+c_{2}\right) \\
y=\beta_{1}\left(a_{1} x+b_{1}\right)+\beta_{2}\left(a_{3} x^{3}+b_{3} x^{2}+c_{3} x+d_{3}\right) \\
y=\gamma_{1}\left(a_{1} x+b_{1}\right)+\gamma_{2}\left(a_{2} x^{2}+b_{2} x+c_{2}\right) \\
+\gamma_{3}\left(a_{3} x^{3}+b_{3} x^{2}+c_{3} x+d_{3}\right)
\end{gathered}
$$

as the combination of linear and $2^{\text {nd }}$ order non-linear and $3^{\text {rd }}$ order non-linear function. Here, $\alpha_{2}=1-\alpha_{1}, \beta_{2}=1-\beta_{1}$, $\gamma_{3}=1-\left(\gamma_{1}+\gamma_{2}\right)$. Comparative discussion concerning (21), (22) and (23) are described in section 5.

\section{Monthly Ratio ${ }^{[5]}$}

For example, if there is the monthly data of $\mathrm{L}$ years as stated bellow:

$$
\left\{x_{i j}\right\}(i=1, \cdots, L)(j=1, \cdots, 12)
$$

Where, $x_{i j} \in R$ in which $j$ means month and $i$ means year and $x_{i j}$ is a shipping data of $\mathrm{i}$-th year, $\mathrm{j}$-th month. Then, monthly ratio $\tilde{x}_{j}(j=1, \cdots, 12)$ is calculated as follows.

$$
\tilde{x}_{j}=\frac{\frac{1}{L} \sum_{i=1}^{L} x_{i j}}{\frac{1}{L} \cdot \frac{1}{12} \sum_{i=1}^{L} \sum_{j=1}^{12} x_{i j}}
$$

\section{Forecasting The Production Data}

\section{A. Analysis Procedure}

Sum total data of production data of X-ray image intensifier tube device and Diagnostic X-ray image processing apparatus from January 2010 to December 2012 are analyzed. These data are obtained from the Annual Report of Statistical Investigation on Statistical-Survey-on-Trends-inPharmaceutical-Production by Ministry of Health, Labour and Welfare in Japan.

The original data are accumulated for X-ray image intensifier tube device data and Diagnostic X-ray image processing apparatus data.

Analysis procedure is as follows. There are 36 monthly data for each case. We use 24 data (1 to 24) and remove trend by the method stated in 2.2 . Then we calculate monthly ratio 
by the method stated in 2.3. After removing monthly trend, the method stated in 2 is applied and Exponential Smoothing Constant with minimum variance of forecasting error is estimated. Then 1 step forecast is executed. Thus, data is shifted to 2nd to 25th and the forecast for 26th data is executed consecutively, which finally reaches forecast of 36th data. To examine the accuracy of forecasting, variance of forecasting error is calculated for the data of 25 th to 36 th data. Final forecasting data is obtained by multiplying monthly ratio and trend. Forecasting error is expressed as:

$$
\begin{gathered}
\varepsilon_{i}=\hat{x}_{i}-x_{i} \\
\bar{\varepsilon}=\frac{1}{N} \sum_{i=1}^{N} \varepsilon_{i}
\end{gathered}
$$

Variance of forecasting error is calculated by:

$$
\sigma_{\varepsilon}^{2}=\frac{1}{N-1} \sum_{i=1}^{N}\left(\varepsilon_{i}-\bar{\varepsilon}\right)^{2}
$$

\section{B. Trend Removing}

Trend is removed by dividing original data by,(21),(22),(23). The patterns of trend removal are exhibited in Table 1.

TABLE I. THE PATTERNS OF TREND REMOVAL

\begin{tabular}{|l|l|}
\hline Pattern 1 & $\alpha_{1}, \alpha_{2}$ are set 0.5 in the equation (21) \\
\hline Pattern 2 & $\beta_{1}, \beta_{2}$ are set 0.5 in the equation (22) \\
\hline Pattern3 & $\alpha_{1}$ is shifted by 0.01 increment in (21) \\
\hline Pattern4 & $\beta_{1}$ is shifted by 0.01 increment in (22) \\
\hline Pattern5 & $\gamma_{1}$ and $\gamma_{2}$ are shifted by 0.01 increment in $(23)$ \\
\hline
\end{tabular}

In pattern 1 and 2 , the weight of $\alpha_{1}, \alpha_{2}, \beta_{1}, \beta_{2}$ are set 0.5 in the equation $(21),(22)$. In pattern 3 , the weight of $\alpha_{1}$ is shifted by 0.01 increment in (21) which satisfy the range $0 \leq \alpha_{1} \leq 1.00$. In pattern 4 , the weight of $\beta_{1}$ is shifted in the same way which satisfy the range $0 \leq \beta_{1} \leq 1.00$. In pattern5, the weight of $\gamma_{1}$ and $\gamma_{2}$ are shifted by 0.01 increment in (23) which satisfy the range $0 \leq \gamma_{1} \leq 1.00,0 \leq \gamma_{2} \leq 1.00$.The best solution is selected which minimizes the variance of forecasting error.

\section{Removing trend of monthly ratio}

After removing trend, monthly ratio is calculated by the method stated in 2.3.

\section{Estimation of Smoothing Constant with Minimum Variance of Forecasting Error}

After removing monthly trend, Smoothing Constant with minimum variance of forecasting error is estimated utilizing (16). There are cases that we cannot obtain a theoretical solution because they do not satisfy the condition of (15).
In those cases, Smoothing Constant with minimum variance of forecasting error is derived by shifting variable from 0.01 to 0.99 with 0.01 interval.

The intermittent demand data often include 0 data. If there are so many 0 data, there is a case we cannot calculate the theoretical solation of smoothing constant.

In that case, we add very tiny data which is not 0 but close to 0 that does not affect anything in calculating parameters (i.e. negligible small).

\section{E. Forecasting AND Variance of Forecasting Error}

Utilizing smoothing constant estimated in the previous section, forecasting is executed for the data of 25th to 36th

data. Final forecasting data is obtained by multiplying monthly ratio and trend. Variance of forecasting error is calculated by (27).

As we have made accumulated data case and tiny data close to 0 added case, we have the following cases altogether.

1. Non Monthly Trend Removal

(1) Accumulated Data

(2) Non Accumulated Data

(2-1) Forecasting from the Accumulated data (Accumulated forecasting data at time $n$-Accumulated data (at time $n-1)$ )

\section{A. Pattern1 B. Pattern2 C. Pattern3 D. Pattern4 E. Pattern5}

(2-2) Forecasting from the tiny data close to 0 added case

A. Pattern1 B. Pattern2 C. Pattern3 D. Pattern4 E. Pattern5

2. Monthly Trend Removal

(1) Accumulated Data

(2) Non Accumulated Data

(2-1) Forecasting from the Accumulated data (Accumulated forecasting data at time $n$-Accumulated data (at time $n-1)$ )

\section{A. Pattern1 B. Pattern2 C. Pattern3 D. Pattern4 E. Pattern5}

(2-2) Forecasting from the tiny data close to 0 added case

\section{A. Pattern1 B. Pattern2 C. Pattern3 D. Pattern4 E. Pattern5}

We can make forecasting by reversely making the data from the forecasting accumulated data, i.e., that is shown at (2-1).

Now, we show them at Figure1 through 6.

Figure 1,2 and 3 show the Non-monthly Trend Removal Case in X-ray image intensifier tube device.

It includes all cases classified above.

Figure 1 shows the Accumulated Data Case in NonMonthly Trend Removal.

Figure 2 shows the Forecasting from the Accumulated Data Case in Non-Monthly Trend Removal. 
Figure 3 shows the Forecasting from the tiny data close to 0 added case in Non-Monthly Trend Removal.

Table 2,3 and 4 show the corresponding variance of forecasting error for each Figure 1,2 and 3.

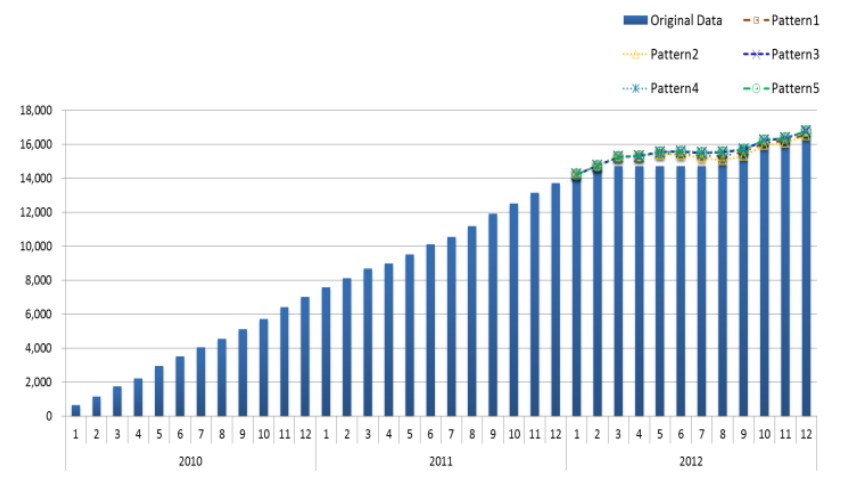

Fig. 1. Forecasting from the Accumulated Data Case in Non-Monthly Trend Removal (1-(1))

TABLE II. VARIANCE OF FORECASTING ERROR (1-(1))

\begin{tabular}{|c|c|c|c|c|}
\hline Pattern1 & Pattern2 & Pattern3 & Pattern4 & Pattern5 \\
\hline 582758.5 & 553598.6 & 655627.9 & 672911.8 & 655627.9 \\
794 & 015 & 015 & 873 & 015 \\
\hline
\end{tabular}

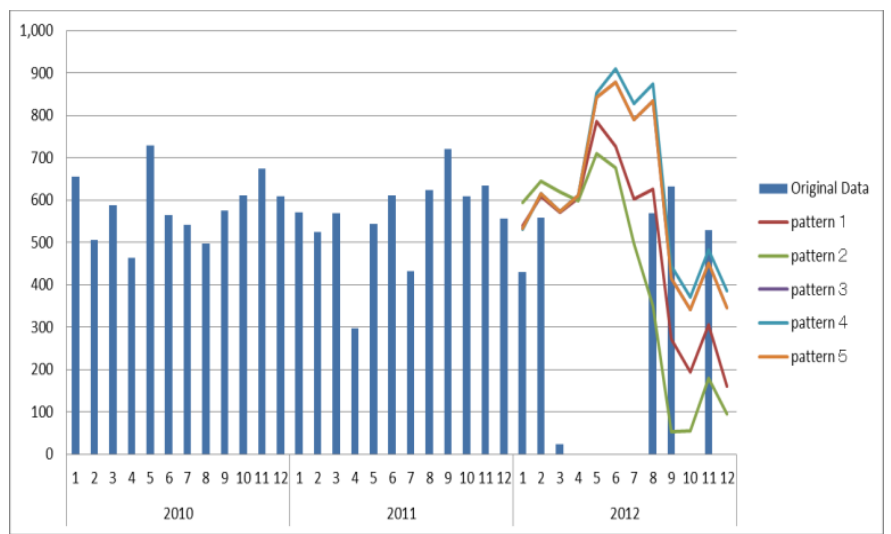

Fig. 2. Forecasting from the Accumulated Data Case in Non-monthly Trend Removal (1-(2-1))

TABLE III. VARIANCE OF FORECASTING ERROR (1-(2-1))

\begin{tabular}{|c|c|c|c|c|}
\hline Pattern1 & Pattern2 & Pattern3 & Pattern4 & Pattern5 \\
\hline 140940.0 & 183864.2 & 134016.6 & 134302.4 & 134016.6 \\
69 & 572 & 212 & 864 & 212 \\
\hline
\end{tabular}

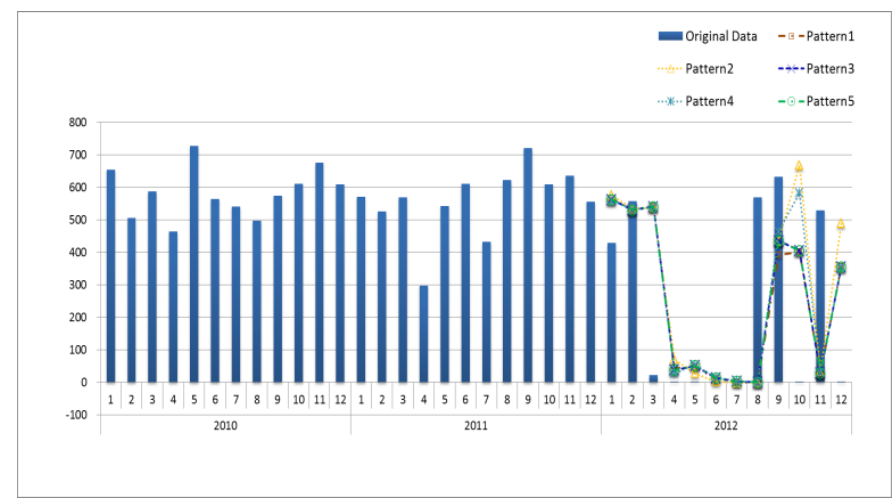

Fig. 3. Forecasting from the Tiny Data close to 0 Added case in NonMonthly Trend Removal (1-(2-2))

TABLE IV. VARIANCE OF FORECASTING ERROR (1-(2-2))

\begin{tabular}{|c|c|c|c|c|}
\hline Pattern1 & Pattern2 & Pattern3 & Pattern4 & Pattern5 \\
\hline 110237.5 & 141410.2 & 108230.8 & 121685.9 & 108230.8 \\
688 & 624 & 417 & 633 & 417 \\
\hline
\end{tabular}

Next, we see the Monthly Trend Removal case.

Figure 4,5 and 6 show the Monthly Trend Removal Case in X-ray image intensifier tube device.

It includes all cases classified above.

Figure 4 shows the Accumulated Data Case in Monthly Trend Removal.

Figure 5 shows the Forecasting from the Accumulated Data Case in Monthly Trend Removal.

Figure 6 shows the Forecasting from the tiny data close to 0 added case in Monthly Trend Removal.

Table 5,6 and 7 show the corresponding variance of forecasting error for each Figure 4,5 and 6.

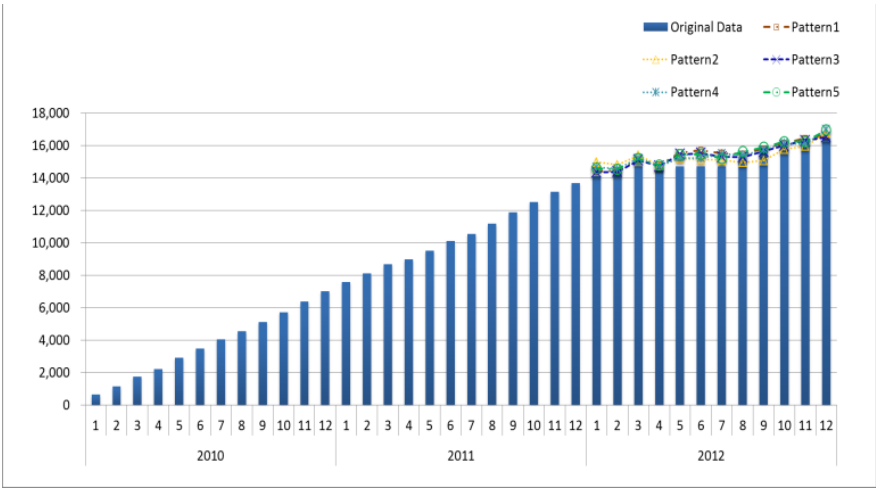

Fig. 4. Accumulated Data case in Monthly Trend Removal (2-(1)) 
TABLE V. VARIANCE OF FORECASTING ERROR (2-(1))

\begin{tabular}{|c|c|c|c|c|}
\hline Pattern1 & Pattern2 & Pattern3 & Pattern4 & Pattern5 \\
\hline 657839.2 & 505482.4 & 621118.7 & 636984.8 & 655587.0 \\
024 & 188 & 598 & 635 & 73 \\
\hline
\end{tabular}

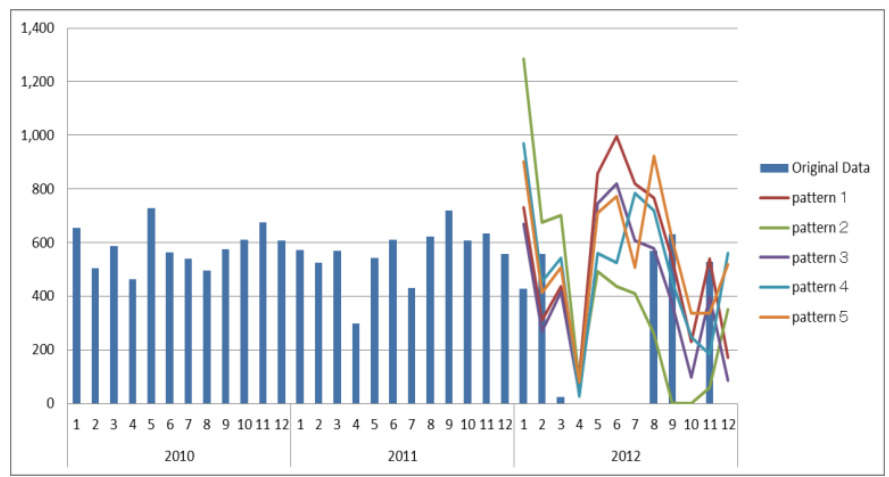

Fig. 5. Forecasting from the accumulated Data case in Monthly Trend Removal (2-(2-1))

TABLE VI. VARIANCE OF FORECASTING ERROR (2-(2-1))

\begin{tabular}{|c|c|c|c|c|}
\hline Pattern1 & Pattern2 & Pattern3 & Pattern4 & Pattern5 \\
\hline 153367.9 & 210960.3 & 139068.3 & 129896.7 & 103268.0 \\
683 & 255 & 784 & 705 & 731 \\
\hline
\end{tabular}

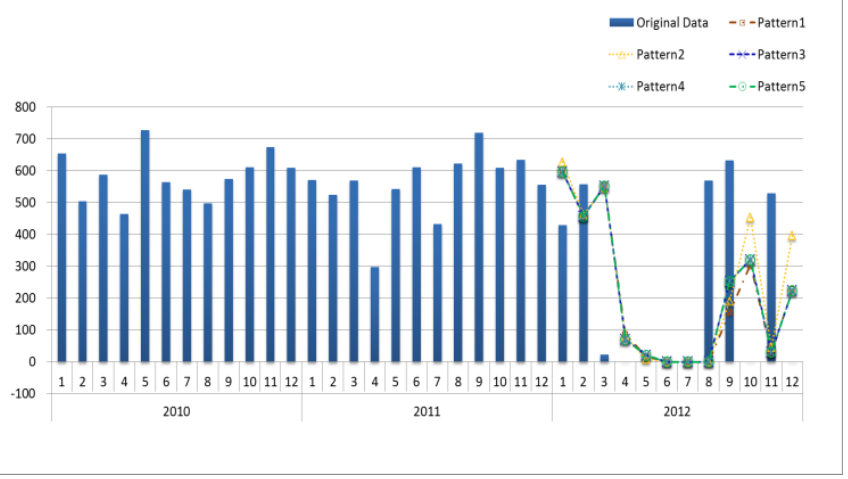

Fig. 6. Forecasting from the Tiny Data close to 0 Added case in Monthly Trend Removal (2-(2-2))

TABLE VII. VARIANCE OF FORECASTING ERROR (2-(2-2))

\begin{tabular}{|c|c|c|c|c|}
\hline Pattern1 & Pattern2 & Pattern3 & Pattern4 & Pattern5 \\
\hline 115474.9 & 131347.7 & 108078.5 & 108078.5 & 108078.5 \\
994 & 067 & 346 & 346 & 346 \\
\hline
\end{tabular}

Table 8 shows the summary for X-ray image intensifier tube devic by the Variance of forecasting error.

TABLE VIII. SUMMARY FOR X-RAY IMAGE INTENSIFIER TUBE DEVICE

\begin{tabular}{|l|l|l|l|l|l|l|l|}
\hline & & \multicolumn{3}{|c|}{ Monthly Trend Removal } & \multicolumn{3}{c|}{ Non Monthly Trend Removal } \\
\hline Name & $\begin{array}{c}\text { X-ray image } \\
\text { intensifie } \\
\text { tube device }\end{array}$ & $\begin{array}{l}\text { Accumulate } \\
\text { d Data }\end{array}$ & $\begin{array}{l}\text { Forecasting } \\
\text { Value }- \\
\text { Accumulate } \\
\text { d Value }\end{array}$ & $\begin{array}{l}\text { Tiny data } \\
\text { close to 0 } \\
\text { added case }\end{array}$ & $\begin{array}{l}\text { Accumulate } \\
\text { d Data }\end{array}$ & $\begin{array}{l}\text { Forecasting } \\
\text { Value }- \\
\text { Accumulate } \\
\text { d Value }\end{array}$ & $\begin{array}{l}\text { Tiny data } \\
\text { close to 0 } \\
\text { added case }\end{array}$ \\
\hline $\begin{array}{c}\text { Minimum variance of } \\
\text { Forecasting Error }\end{array}$ & 505482.4188 & 103268.0731 & 108078.5346 & 553598.6015 & 134016.6212 & 108230.8417 \\
\hline
\end{tabular}

Now, we proceed to the case of Diagnostic X-ray image processing apparatus. Figure 7,8 and 9 show the Non-monthly Trend Removal Case in Diagnostic X-ray image processing apparatus.

It includes all cases classified above.

Figure 7 shows the Accumulated Data Case in NonMonthly Trend Removal.

Figure 8 shows the Forecasting from the Accumulated Data Case in Non-Monthly Trend Removal.

Figure 9 shows the Forecasting from the tiny data close to 0 added case in Non-Monthly Trend Removal.

Table 9,10 and 11 show the corresponding variance of forecasting error for each Figure 7,8 and 9.

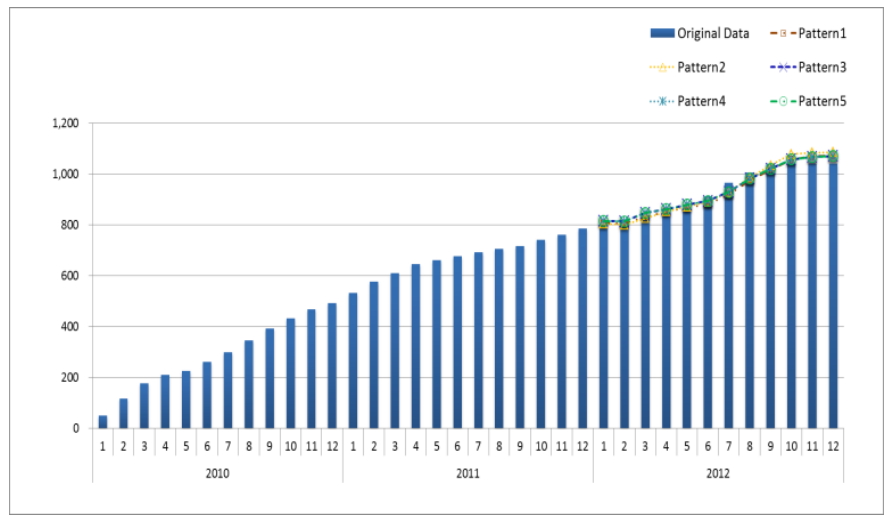

Fig. 7. Accumulated Data case in Non-Monthly Trend Removal (1-(1))

TABLE IX. VARIANCE OF FORECASTING ERROR (1-(1))

\begin{tabular}{|c|c|c|c|c|}
\hline Pattern1 & Pattern2 & Pattern3 & Pattern4 & Pattern5 \\
\hline 12144.26 & 13893.64 & 11033.83 & 11033.83 & 11033.83 \\
114 & 686 & 677 & 677 & 677 \\
\hline
\end{tabular}

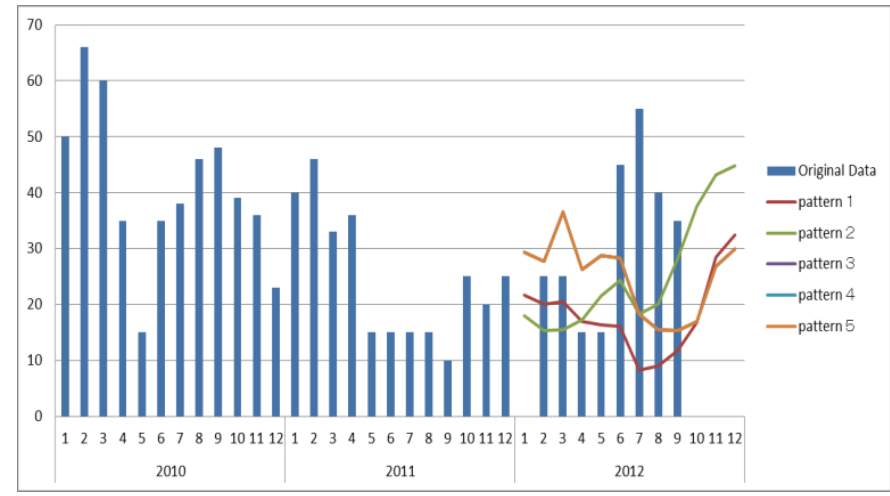

Fig. 8. Forecasting from the accumulated Data case in Non-Monthly Trend Removal (1-(2-1))

TABLE X. VARIANCE OF FORECASTING ERROR (1-(2-1))

\begin{tabular}{|c|c|c|c|c|}
\hline Pattern1 & Pattern2 & Pattern3 & Pattern4 & Pattern5 \\
\hline 642.6679 & 714.8801 & 515.2469 & 515.2469 & 515.2469 \\
766 & 978 & 097 & 097 & 097 \\
\hline
\end{tabular}




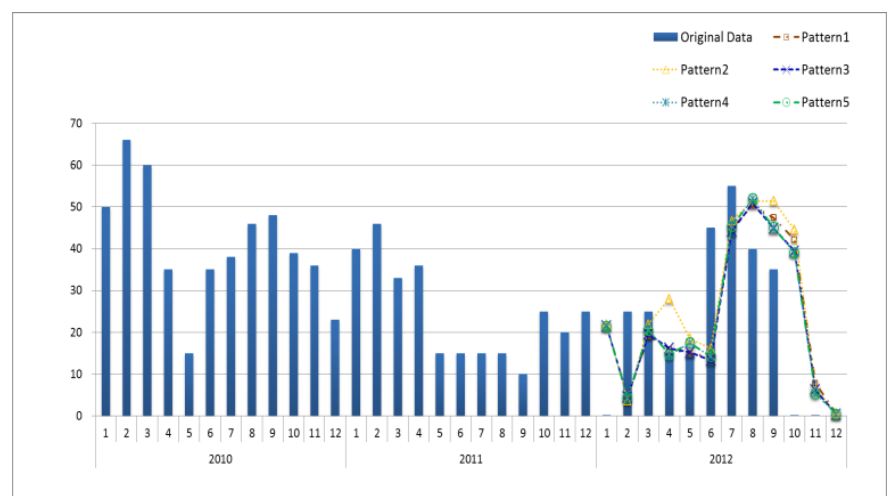

Fig. 9. Forecasting from the Tiny Data close to 0 Added case in NonMonthly Trend Removal (1-(2-2))

TABLE XI. VARIANCE OF FORECASTING ERROR (1-(2-2))

\begin{tabular}{|c|c|c|c|c|}
\hline Pattern1 & Pattern2 & Pattern3 & Pattern4 & Pattern5 \\
\hline 365.3419 & 381.0283 & 345.9402 & 336.4640 & 336.4640 \\
523 & 782 & 412 & 944 & 944 \\
\hline
\end{tabular}

Next, we see the Monthly Trend Removal case.

Figure 10,11 and 12 show the Monthly Trend Removal Case in Diagnostic X-ray image processing apparatus.

It includes all cases classified above.

Figure 10 shows the Accumulated Data Case in Monthly Trend Removal.

Figure 11 shows the Forecasting from the Accumulated Data Case in Monthly Trend Removal.

Figure 12 shows the Forecasting from the tiny data close to 0 added case in Monthly Trend Removal.

Table 12,13 and 14 show the corresponding variance of forecasting error for each Figure 10,11 and 12.

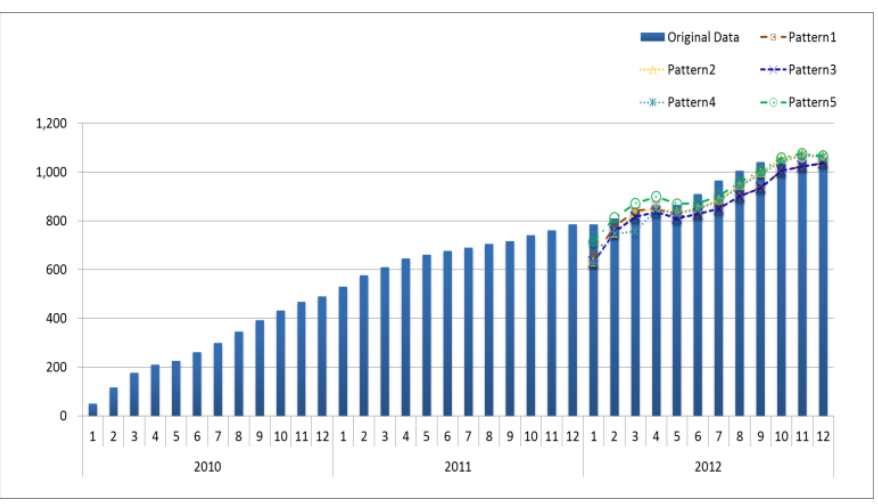

Fig. 10. Accumulated Data case in Monthly Trend Removal (2-(1))

TABLE XII. VARIANCE OF FORECASTING ERROR (2-(1))

\begin{tabular}{|c|c|c|c|c|}
\hline Pattern1 & Pattern2 & Pattern3 & Pattern4 & Pattern5 \\
\hline 13726.72 & 19516.74 & 15344.88 & 20527.68 & 13453.32 \\
311 & 892 & 764 & 403 & 873 \\
\hline
\end{tabular}

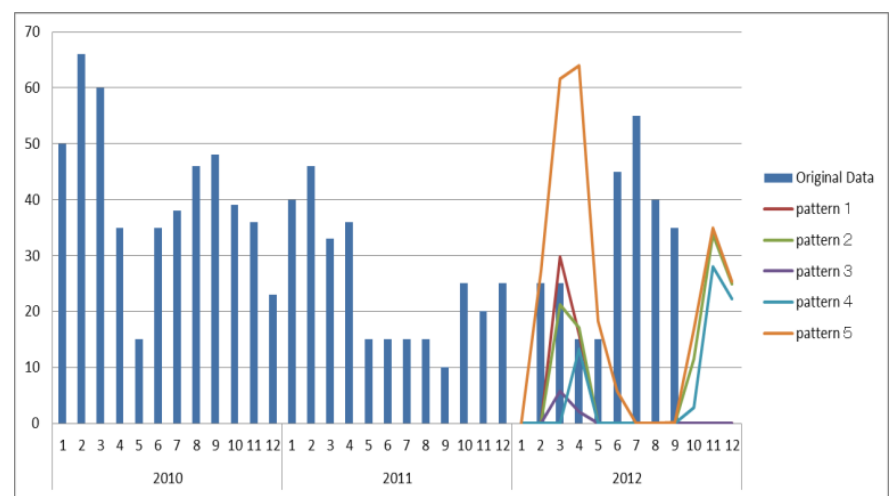

Fig. 11. Forecasting from the accumulated Data case in Monthly Trend Removal (2-(2-1))

TABLE XIII. VARIANCE OF FORECASTING ERROR (2-(2-1))

\begin{tabular}{|c|c|c|c|c|}
\hline Pattern1 & Pattern2 & Pattern3 & Pattern4 & Pattern5 \\
\hline 463.52944 & 804.97669 & 379.28200 & 697.531 & 1206.97 \\
54 & 78 & 22 & 27 & 5423 \\
\hline
\end{tabular}

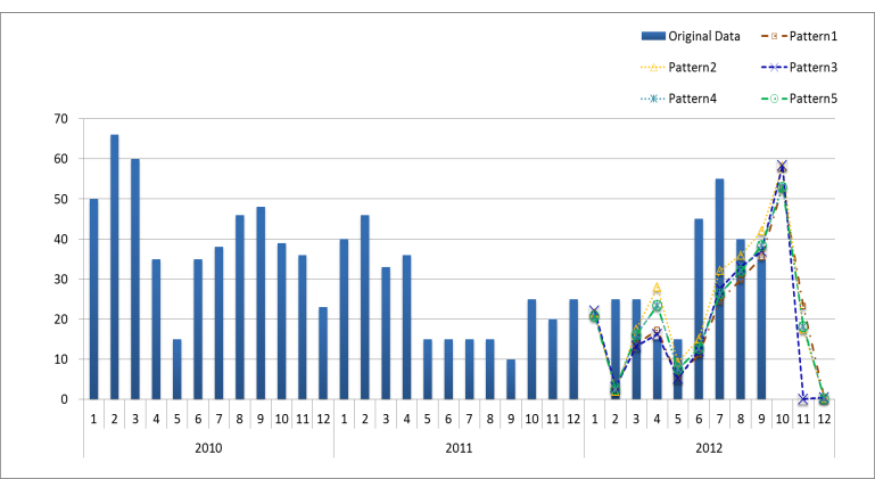

Fig. 12. Forecasting from the Tiny Data close to 0 Added case in Monthly Trend Removal (2-(2-2))

TABLE XIV. VARIANCE OF FORECASTING ERROR (2-(2-2))

\begin{tabular}{|c|c|c|c|c|}
\hline Pattern1 & Pattern2 & Pattern3 & Pattern4 & Pattern5 \\
\hline 603.303 & 579.916 & 585.7549 & 565.5003 & 565.5003 \\
7984 & 3939 & 099 & 321 & 321 \\
\hline
\end{tabular}

Table 17 shows the summary for Diagnostic X-ray image processing apparatus by the Variance of forecasting error.

TABLE XV. SUMMARY FOR DIAGNOSTIC X-RAY IMAGE PROCESSING APPARATUS

\begin{tabular}{|c|l|l|l|l|l|l|l|}
\hline & & \multicolumn{3}{|c|}{ Monthly Trend Removal } & \multicolumn{3}{|c|}{ Non Monthly Trend Removal } \\
\hline Name & $\begin{array}{l}\text { Diagnostic } \\
\text { X-ray image } \\
\text { processing } \\
\text { apparatus of } \\
\text { other }\end{array}$ & $\begin{array}{l}\text { Accumulate } \\
\text { d Data }\end{array}$ & $\begin{array}{l}\text { Forecasting } \\
\text { Value }- \\
\text { Accumulate } \\
\text { d Value }\end{array}$ & $\begin{array}{l}\text { Tiny data } \\
\text { close to 0 } \\
\text { added case }\end{array}$ & $\begin{array}{l}\text { Accumulate } \\
\text { d Data }\end{array}$ & $\begin{array}{l}\text { Forecasting } \\
\text { Value }- \\
\text { Accumulate } \\
\text { d Value }\end{array}$ & $\begin{array}{l}\text { Tiny data } \\
\text { close to 0 } \\
\text { added case }\end{array}$ \\
\hline $\begin{array}{l}\text { Minimum variance of } \\
\text { Forecasting Error }\end{array}$ & 13453.32873 & 379.2820022 & 565.5003321 & 11033.83677 & 515.2469097 & 336.4640944 \\
\hline
\end{tabular}

IV. Discussion

In the case of X-ray image intensifier tube device, Monthly Trend Removal case was better than Non-Monthly Trend 
Removal case. This time series had a rather clear monthly trend and the result had reflected them. Forecasting from the accumulated data case (2-(2-1)) was better than those of the tiny data close to 0 added case (2-(2-2)) in this Monthly Trend Removal case for X-ray image intensifier tube device. On the other hand, in the case of Diagnostic X-ray image processing apparatus, Non-Monthly Trend Removal case was better than Monthly Trend Removal case. The time series of Diagnostic $\mathrm{X}$-ray image processing apparatus does not have clear monthly trend. Forecasting from the tiny data close to 0 added case (1(2-2)) was better than those of Accumulated data case (1-(21)). By the way, forecasting of accumulated data (1-(1), 2-(1)) shows rather good result. It can be used as one of the tool to decide when and how much volume to procure the materials etc.. It can be utilized as a new method to procure in supply chain management.

\section{CONCLUSION}

The needs for intermittent demand forecasting are increasing. In this paper, a new method for cumulative forecasting method was proposed. The data was cumulated and to this cumulated time series, the new method was applied to improve the forecasting accuracy. The forecasting result was compared with those of the non-cumulative forecasting method. The new method shows that it is useful for the forecasting of intermittent demand data. Forecasting from the accumulated data case (2-(2-1)) was better than those of the tiny data close to 0 added case (2-(2-2)) in this Monthly Trend Removal case for X-ray image intensifier tube device. On the other hand, in the case of Diagnostic X-ray image processing apparatus, forecasting from the tiny data close to 0 added case (1-(2-2)) was better than those of Accumulated data case (1(2-1)). Among them, forecasting of accumulated data (1-(1), 2-(1)) shows rather good result. It can be used as one of the tool to decide when and how much volume to procure the materials etc.. It can be utilized as a new method to procure in supply chain management.

\section{FUTURE WORKS}

It is our future works to investigate much further cases to confirm the effectiveness of our new method. The effectiveness of this method should be examined in various cases.

\section{REFERENCES}

[1] Box, G..E.P., Jenkins, G.M.\& Reinsel, G.C.,Time Series analysis: forecasting and control, Wiley, $4^{\text {th }}$ edn.,2008.

[2] Lydia Shenstone and Rob J. Hyndma, "Stochastic models underlying Croston's method for intermittent demand forecasting", Journal of Forecasting, 24:389-402,2005.

[3] Kenji Tanaka, Yukihiro Miyamura and Jing Zhang, "The Cluster Grouping Approach of Sales Forecasting Model for Book Publishing", International Journal of Japan Association for Management Systems, Vol.4, No.1,pp.31-35,2012.

[4] Nguyen Khoa Viet Froung, Shin Sangmun, Vo Thanh Nha, Kwon Ichon, "Intermittent Demand forecasting by using Neural Network with simulated data", Proceedings of the 2011 International Engineering and Operations Management Kuala Lumpur, Malasia, pp.723-728,2011.

[5] Kazuhiro Takeyasu and Keiko Nagata, "Estimation of Smoothing Constant of Minimum Variance with Optimal Parameters of Weight", International Journal of Computational Science Vol.4,No.5, pp. 411425,2010 